\title{
A TWO-FRACTAL OVERLAP MODEL OF EARTHQUAKES
}

\author{
Bikas K Chakrabarti and Arnab Chatterjee \\ (bikask.chakrabarti@saha.ac.in, arnab.chatterjee@saha.ac.in) \\ Theoretical Condensed Matter Physics Division and \\ Centre for Applied Mathematics and Computational Science, \\ Saha Institute of Nuclear Physics, 1/AF Bidhannagar, Kolkata 700064, India
}

\begin{abstract}
We introduce here the two-fractal model of earthquake dynamics. As the fractured surfaces have self-affine properties, we consider the solid-solid interface of the earth's crust and the tectonic plate below as fractal surfaces. The overlap or contact area between the two surfaces give a measure of the stored elastic energy released during a slip. The overlap between two fractals change with time as one moves over the other and we show that the time average of the overlap distribution follows a Gutenberg-Richter like power-law, with similar exponent value.
\end{abstract}

Key words: earthquake, fractals, Cantor sets, Gutenberg-Richter law

\section{Introduction}

The earth's solid outer crust, about 20 kilometers in average thickness, rests on the tectonic shells. Due to the high temperature-pressure phase changes and the consequent powerful convective flow in the earth's mantle (a fluid of very high density), at several hundreds of kilometers of depth, the tectonic shell, divided into a small number (about ten) of mobile plates, has relative velocities of the order of a few centimeters per year (Gutenberg and Richter, 1954; Kostrov and Das, 1989; Scholz, 1990). Over several tens of years, enormous elastic strains develop on the earth's crust when sticking (due to the solid-solid friction) to the moving tectonic plate. When sudden slips occur between the crust and the tectonic plate, these stored elastic energies are released in 'bursts', causing the damages during the earthquakes.

Earthquakes occur due to fault dynamics in the lithosphere. A geological fault is created by a fracture in the rock layers, and is comprised of the rock surfaces in contact. The two parts of the fault are in very slow relative motion which causes the surfaces to slide. Because of the uniform motion of the tectonic plates, the elastic strain energy stored in a portion of the crust (block), moving with the plate relative to a 'stationary' neighboring part of the crust, can vary only due to the random strength of the solid-solid friction between the crust and the plate. A slip

(C) 2018 Springer. Printed in the Netherlands. 
occurs when the accumulated stress exceeds the resistance due to the frictional force. The potential energy of the strain is thereby released, causing an earthquake. As mentioned before, the observed distribution of the elastic energy release in various earthquakes seems to follow a power law.

The slip is eventually stopped by friction and stress starts developing again. Strain continues to develop till the fault surfaces again slip. This intermittent stickslip process is the essential characteristic feature of fault dynamics. The overall distribution of earthquakes, including main shocks, foreshocks and aftershocks, is given by the Gutenberg-Richter law (Gutenberg and Richter, 1954; Gutenberg and Richter, 1944):

$$
\log _{10} \operatorname{Nr}(\mathcal{M}>M)=a-b M
$$

where, $\operatorname{Nr}(\mathcal{M}>M)$ denotes the number (or, the frequency) of earthquakes of magnitudes $\mathcal{M}$ that are greater than a certain value $M$. The constant $a$ represents the total number of earthquakes of all magnitudes: $a=\log _{10} \operatorname{Nr}(\mathcal{M}>0)$ and the value of the coefficient $b$ is presumed to be universal. In an alternative form, the Gutenberg-Richter law is expressed as a relation for the number (or, the frequency) of earthquakes in which the energy released $\mathcal{E}$ is greater than a certain value $E$ :

$$
\operatorname{Nr}(\mathcal{E}>E) \sim E^{-b / \beta} \text {, suggesting } \operatorname{Nr}(E) \sim E^{-\gamma},
$$

for the number density of earthquakes, where $\gamma=1+b / \beta$. The value of the exponent $\gamma$ is generally observed to be around unity (Knopoff, 2000); see also http://web.cz3.nus.edu.sg/ ${ }^{\sim}$ chenk/gem2503_3/notes7_1.htm.

One class of models for simulating earthquakes is based on the collective motion of an assembly of connected elements that are driven slowly, of which the block-spring model due to Burridge and Knopoff (Burridge and Knopoff, 1967 ) is the prototype. The Burridge-Knopoff model and its variants (Carlson et al, 1994; Olami et al., 1992) have the stick-slip dynamics necessary to produce earthquakes. The underlying principle in this class of models is self-organized criticality (Bak, 1997).

Another class of models for simulating earthquakes is based on overlapping fractals, which will be discusses in details in the next sections.

\section{Fractals}

A fractal is a geometrical object that displays self-similarity on all scales. For random fractals, the object need not exhibit exactly the same structure at all scales, but the same 'type' of structures appear on all scales. For example, the Black sea coastline measured with different length rulers will show differences: the shorter the ruler, the longer the 'length' measured but not exactly following the ratio of the inverse of the lengths of the rulers; greater than that! This is because more structures come into play at lower length scales; or in other words, the coastline is 


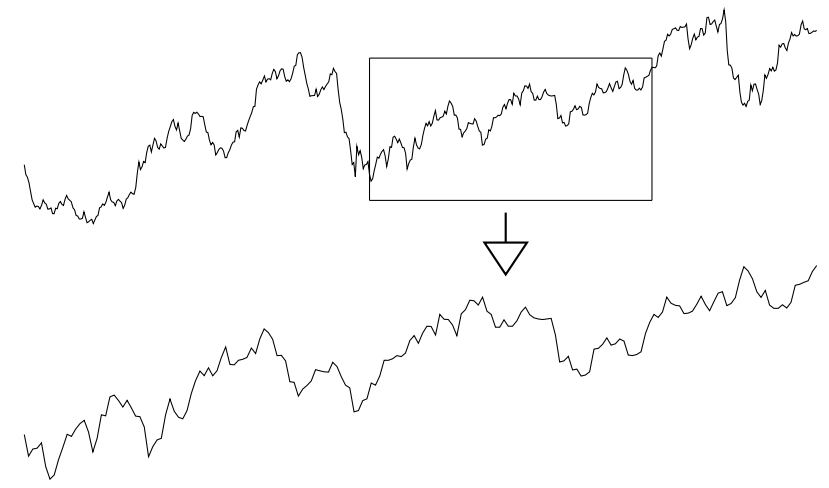

Figure 1. Fractal structure of Black sea coastline at Işik (Istanbul), represented actually by a time series of stock price. This shows the remarkable self-similarity (examplified by the blow up of the segment in the box) involved in many such natural processes.

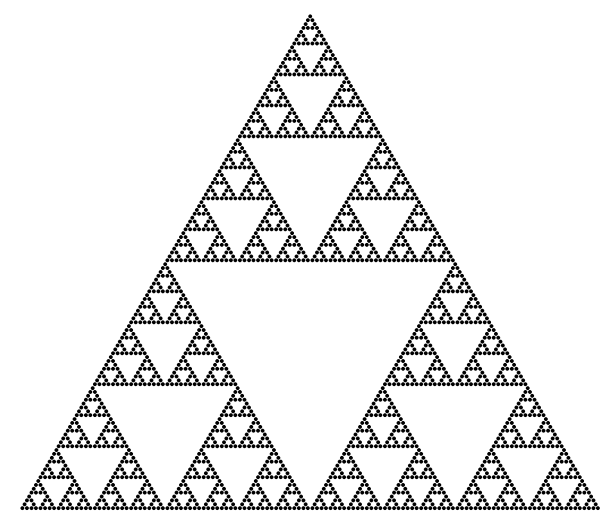

Figure 2. Sierpinski gasket (at generation number $n=5$ ), having fractal dimension $\log 3 / \log 2$ (when $n \rightarrow \infty$ ).

not really a 'line' but rather a fractal having dimension greater than unity. Looking at smaller and smaller length-scales, one can find self-similar structures (see Fig. 1).

One can easily construct such regular fractals as carpets or gaskets (see Fig. 2). In Fig 2, a basic unit of equilateral triangle with an inner triangle obtained by joining the mid-points of each side and keeping the inner space void, one constructs a gasket. At each step, as the length of each side changes by a factor $L=2$, the mass of the fractal changes by a factor $M\left(=L^{d_{f}}\right)=3$, giving therefore the fractal dimension of the object to be $d_{f}=\log 3 / \log 2$. This object in Fig. 2 of course represents a non-random fractal. However, a random fractal (as in Fig. 1) can be easily constructed if the void is not always at the center but at any of the 4 triangles at random for each generation; the (mass) dimension $d_{f}$ remain 


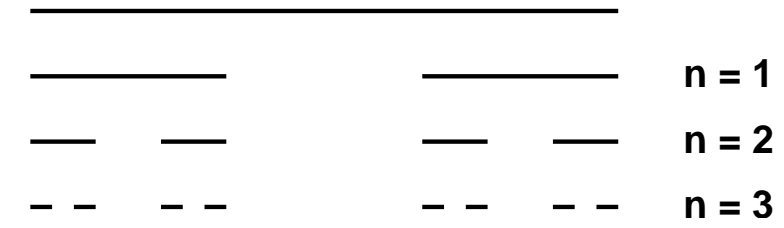

Figure 3. Construction process of Cantor set is shown (upto $n=3$ ). The set becomes a fractal when $n \rightarrow \infty$.

the same. A similar fractal of dimension $\log 2 / \log 3$ can be constructed as the Cantor set shown in Fig. 3. Starting from a set of all real numbers from 0 to 1 one removes the subset from the middle third, so that for each $l=3$, the mass (size) of the set $m=2$ and hence the dimension as $n \rightarrow \infty$. This void subset can again be randomly chosen, giving a random Cantor set, with same $d_{f}$.

\section{Fractal overlap model of earthquake}

Overlapping fractals form a whole class of models to simulate earthquake dynamics. These models are motivated by the observation that a fault surface, like a fractured surface (Chakrabarti and Benguigui, 1997), is a fractal object (Okubo and Aki, 1987; Scholz and Mandelbrot, 1989; Sahimi, 1993). Consequently a fault may be viewed as a pair of overlapping fractals. Fractional Brownian profiles have been commonly used as models of fault surfaces (Brown and Scholz, 1985; Sahimi, 1993). In that case the dynamics of a fault is represented by one Brownian profile drifting on another and each intersection of the two profiles corresponds to an earthquake (De Rubeis et al, 1996). However the simplest possible model of a fault - from the fractal point of view - was proposed by Chakrabarti and Stinchcombe (Chakrabarti and Stinchcombe, 1999). This model is a schematic representation of a fault by a pair of dynamically overlapping Cantor sets. It is not realistic but, as a system of overlapping fractals, it has the essential feature. Since the Cantor set is a fractal with a simple construction procedure, it allows us to study in detail the statistics of the overlap of one fractal object on another. The two fractal overlap magnitude changes in time as one fractal moves over the other. The overlap (magnitude) time series can therefore be studied as a model time series of earthquake avalanche dynamics (Carlson et al, 1994).

The statistics of overlaps between two fractals is not studied much yet, though their knowledge is often required in various physical contexts. It has been established recently that since the fractured surfaces have got well-characterized self-affine properties, the distribution of the elastic energies released during the slips between two fractal surfaces (earthquake events) may follow the overlap distribution of two self-similar fractal surfaces (Chakrabarti and Stinchcombe, 1999; Pradhan et al, 2003). Chakrabarti and Stinchcombe (Chakrabarti and Stinchcombe, 


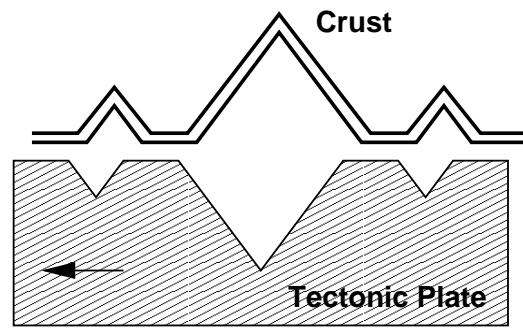

(a)

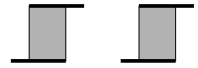

(b)

Figure 4. (a) Schematic representations of a portion of the rough surfaces of the earth's crust and the supporting (moving) tectonic plate. (b) The one dimensional projection of the surfaces form Cantor sets of varying contacts or overlaps $(s)$ as one surface slides over the other.

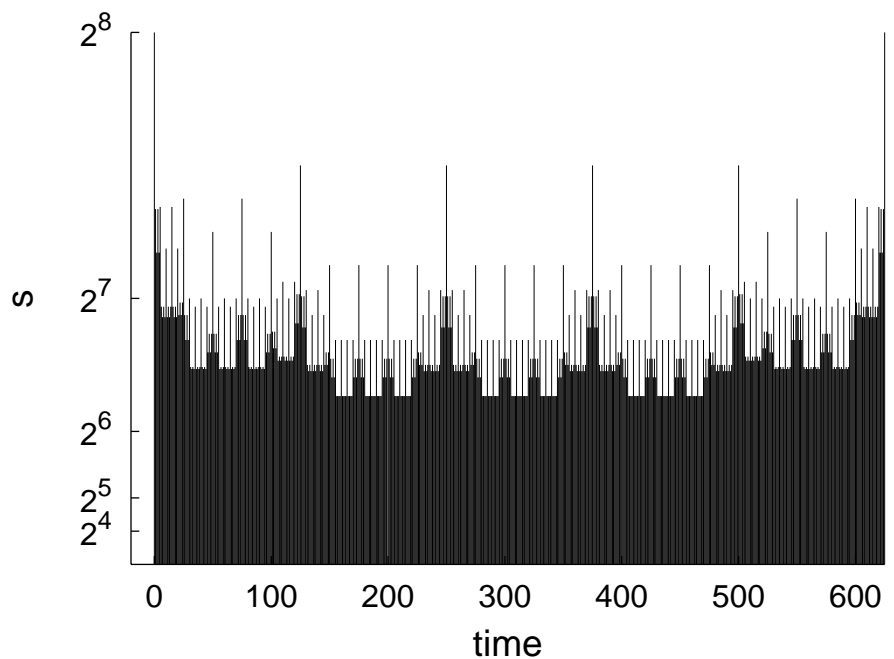

Figure 5. For two Cantor sets (as shown in Fig. 4(b); $n=2$ ), one moving uniformly over the other (periodic boundary conditions), the total measure of the shaded region contribute to the overlap $s$; the time variation of which is shown (for $n=4$ ).

1999) had shown analytically by renormalization group calculations that for regular fractal overlap (Cantor sets and carpets) the contact area distribution $\rho(s)$ follows a simple power law decay:

$$
\rho(s) \sim s^{-\tilde{\gamma}} ; \tilde{\gamma}=1 .
$$

Study of the time $(t)$ variation of contact area (overlap) $s(t)$ between two wellcharacterized fractals having the same fractal dimension as one fractal moves over the other with constant velocity, has revealed some features which can be utilized to predict the 'large events' (Pradhan et al, 2004). 
Bhattacharyya (Bhattacharyya, 2005) has recently studied this overlap distribution for two Cantor sets with periodic boundary conditions and each having dimension $\log 2 / \log 3$. It was shown, using exact counting, that if $s \equiv 2^{n-k}$ ( $n$ is the generation number) then the probability $\tilde{\rho}(s)$ to get an overlap $s$ is given by a binomial distribution (Bhattacharyya, 2005)

$$
\tilde{\rho}\left(2^{n-k}\right)=\left(\begin{array}{c}
n \\
n-k
\end{array}\right)\left(\frac{1}{3}\right)^{n-k}\left(\frac{2}{3}\right)^{k} \sim \exp \left(-r^{2} / n\right) ; r \rightarrow 0,
$$

where $r^{2}=\left[\frac{3}{2}\left(\frac{2}{3} n-k\right)\right]^{2}$. Expressing therefore $r$ by $\log s$ near the maxima of $\tilde{\rho}(s)$, one can again rewrite (4) as

$$
\tilde{\tilde{\rho}}(s) \sim \exp \left(-\frac{(\log s)^{2}}{n}\right) ; n \rightarrow \infty .
$$

Noting that $\tilde{\rho}(s) d(\log s) \sim \rho(s) d s$, we find $\rho(s) \sim s^{-\tilde{\gamma}}, \tilde{\gamma}=1$, as in (3) as the binomial or Gaussian part becomes a very weak function of $s$ as $n \rightarrow \infty$ (Bhattacharyya et al., 2005). It may be noted that this exponent value $\tilde{\gamma}=1$ is independent of the dimension of the Cantor sets considered (here $\log 2 / \log 3$ ) or for that matter, independent of the fractals employed. It also denotes the general validity of (3) even for disordered fractals, as observed numerically (Pradhan et al, 2003; Pradhan et al, 2004).

Identifying the contact area or overlap $s$ between the self-similar (fractal) crust and tectonic plate surfaces as the stored elastic energy $E$ released during the slip, the distribution (3), of which a derivation is partly indicated here, reduces to the Gutenberg-Richter law (2) observed.

\section{Summary}

We introduce here the two-fractal overlap model of earthquake where the average distribution of the overlaps between the surfaces, as one fractal (here, Cantor set) moves over the other gives the Gutenberg-Richter like distribution (3), with $\tilde{\gamma}=1$ exactly in the model. We note that this is an exactly solvable model of earthquake dynamics and the result for the distributions compare favorably with the observations.

\section{Acknowledgements}

We are grateful to P. Bhattacharyya, P. Chaudhuri, M. K. Dey, S. Pradhan, P. Ray and R. B. Stinchcombe for collaborations in various stages of developing this model. 


\section{References}

Bak P. (1997) How Nature Works, Oxford Univ. Press, Oxford.

Bhattacharyya P. (2005) Physica A 348, 199.

Bhattacharyya P., Chatterjee A., and Chakrabarti B. K. (2005) arXiv:physics/0510038.

Brown S. R., and Scholz C. H. (1985) J. Geophys. Res. 90, 12575.

Burridge R., and Knopoff L. (1967) Bull. Seismol. Soc. Am. 57, 341.

Carlson J. M., Langer J. S., and Shaw B. E. (1994) Rev. Mod. Phys. 66, 657.

Chakrabarti B. K., and Benguigui L. G. (1997) Statistical Physics of Fracture and Breakdown in Disorder Systems Oxford Univ. Press, Oxford.

Chakrabarti B. K., and Stinchcombe R. B. (1999) Physica A 270, 27.

De Rubeis V., Hallgass R., Loreto V., Paladin G., Pietronero L., and Tosi P. (1996) Phys. Rev. Lett. 76, 2599.

Gutenberg B., and Richter C. F. (1944) Bull. Seismol. Soc. Am. 34, 185.

Gutenberg B., and Richter C. F. (1954) Seismicity of the Earth, Princeton Univ. Press, Princeton.

Knopoff L. (2000) Proc. Natl. Acad. Sci. USA. 97, 11880.

Kostrov B. V., and Das S. (1990) Principles of Earthquake Source Mechanics, Cambridge Univ. Press, Cambridge.

Okubo P. G., and Aki K. (1987) J. Geophys. Res. 92, 345.

Olami Z., Feder H. J. S., and Christensen K. (1992) Phys. Rev. Lett. 68, 1244.

Pradhan S., Chakrabarti B. K., Ray P., and Dey M. K. (2003) Physica Scripta T106, 77.

Pradhan S., Chaudhuri P., and Chakrabarti B. K. (2004) Continuum Models and Discrete Systems, Bergman D. J., and Inan E. Eds, Nato Sc. Series, Kluwer Academic Publishers, Dordrecht, pp.245-250; cond-mat/0307735.

Sahimi M. (1993) Rev. Mod. Phys. 65, 1393.

Scholz C. H. (1990), The Mechanics of Earthquake and Faulting, Cambridge Univ. Press, Cambridge.

Scholz C. H., and Mandelbrot B. B. Eds. (1989) Fractals in Geophysics, Birkhaüser, Basel. 